\title{
Efecto del Desollado y Desangrado de Anchoveta (Engraulis ringens) en Solución de Citrato Sódico
}

\author{
EfFect of Flaying and Bleeding of Anchovy (Engraulis ringens) in Sodium \\ Citrate Solution \\ Santos Teodoro Maza Ramírez ${ }^{1,3}$, Enzo Aldoradin Puza1, Carlos Daniel Pariona \\ Velarde $^{1}$, Eliud Dennis Arpi Anco ${ }^{1}$, María Úrsula Rosales-Hartshorn ${ }^{2}$
}

\section{Resumen}

La corta duración de la vida útil de la anchoveta congelada influye en la limitada utilización de este producto. En el presente estudio, la anchoveta entera (Engraulis ringens) fue desollada, descabezada, eviscerada y desangrada en agua (Control) y en una solución de $2.5 \%$ citrato sódico (Tratamiento) y posteriormente congelada, glaseada y almacenada a $-25{ }^{\circ} \mathrm{C}$ durante 14 meses. El valor peróxido (VP), acidez (A), perfil de ácidos grasos (PAG), sustancias reactivas al ácido tiobarbitúrico (SRAT), hierro hemínico $(\mathrm{HH}), \mathrm{pH}$ y los atributos sensoriales de las muestras fueron evaluados mensualmente. Los resultados de los análisis de VP, SRAT, A, pH y el análisis sensorial mostraron diferencias significativas $(\mathrm{p}<0.05)$ entre el tratamiento y la muestra control, donde la muestra bajo tratamiento presentó mejores características de conservación. Los valores de $\mathrm{HH}$ y PAG presentaron un comportamiento variable. La vida útil de la anchoveta desollada, descabezada y eviscerada (control y tratamiento) fue de 14 meses.

Palabras clave: citrato sódico, anchoveta, Engraulis ringens, desollado, desangrado, vida útil

\section{AbstraCt}

The short duration of the useful life of frozen anchovy affects the limited use of this product. In the present study the whole anchovy (Engraulis ringens) was flayed, beheaded, gutted and bled in water (Control) and in a solution of $2.5 \%$ sodium citrate (Treatment) and subsequently frozen, glazed and stored at $-25{ }^{\circ} \mathrm{C}$ for 14 months. The peroxide value (PV), acidity (A), fatty acid profile (PAG), thiobarbituric acid (TBARS),

\footnotetext{
${ }^{1}$ Dirección de Investigación, Desarrollo, Innovación y Transferencia Tecnológica (DIDITT), Instituto Tecnológico de la Producción, Callao, Perú

${ }^{2}$ Washington State University, EEUU

${ }^{3}$ E-mail: smaza@itp.gob.pe
}

Recibido: 17 de febrero de 2016

Aceptado para publicación: 19 de mayo de 2016 
heme iron $(\mathrm{HH}), \mathrm{pH}$ and sensory attributes of the samples were evaluated monthly. The results of the analysis of VP, TBARS, A, $\mathrm{pH}$ and sensory analysis showed significant differences $(\mathrm{p}<0.05)$ between treatment and control sample, where the sample under treatment showed better conservation features. HH values and PAG had a variable behaviour. The lifespan of the flayed, beheaded and gutted (control and treatment) anchovy was 14 months.

Key words: sodium citrate, anchovy, Engraulis ringens, flay, bleed, lifespan

\section{INTRODUCCIÓN}

Alrededor del 98\% del desembarque de anchoveta Engraulis ringens está dirigido a la producción de harina de pescado (2 $263933 \mathrm{t}$ ), mientras que solo el $0.1 \%$ es para la anchoveta congelada (PRODUCE, 2014). El reducido porcentaje de utilización de anchoveta congelada se debe, entre varios factores, a su limitado periodo de vida útil, siendo 180 días para la anchoveta entera y 150 días para la descabezada y eviscerada (Maza et al., 2008). Esto es consecuencia de la captura en demasía en las bodegas de las embarcaciones, insuficiente uso de hielo, exposición a la luz solar, exposición prolongada a temperatura ambiente antes de su congelación y forma de congelación, entre otros, que evidencian una corta durabilidad durante el almacenamiento a $-25^{\circ} \mathrm{C}$ (Semenov et al., 1979, citado por Karacam y Boran, 1996).

Estos factores también influyen en la conservación de otras especies y condiciones de almacenamiento, tales como refrigerado (Okoro et al., 2010), congelado (Aubourg y Ugliano, 2002; Salas et al., 2004), en hielo (Chávez et al., 2004; Rezaei y Hosseini, 2008) o en mezcla de hielo y agua «cremolada»(Sánchez et al., 2004), que afectan su calidad nutritiva y sensorial, ocasionando el rechazo del consumidor. Estos factores convergen a la continuidad de la utilización de anchoveta en la producción de harina de pescado para la alimentación animal, que es una actividad industrial rentable que no requiere una estricta conservación de la anchoveta.

El desangrado inmediato de los peces vivos retrasa el rigor mortis y el ablandamiento (Richards y Hultin, 2002). La sangre en el tejido muscular induce el olor y sabor desagradable de la carne durante el almacenamiento en hielo debido a la oxidación (Terayama y Yamanaka, 2000). Por otro lado, se ha reportado que el desangrado retarda la oxidación de lípidos de la carne molida de trucha durante el almacenamiento a $2{ }^{\circ} \mathrm{C}$ (Richards y Hultin, 2002).

Se ha demostrado el efecto prooxidante del cloruro sódico en concentraciones de $1 \mathrm{a}$ $2.5 \%$ en productos cárnicos, de allí que el cloruro sódico podría desplazar al hierro hemo del sitio de enlace en las hemoproteínas, mioglobina $(\mathrm{Mg})$ y hemoglobina $(\mathrm{Hb})$ obteniendo hierro libre o hierro no hemo, acelerando el proceso de oxidación (Hamid et al., 2010).

Las hemoproteínas de los peces pelágicos son potentes catalizadores de la oxidación de lípidos en el músculo debido a la liberación del hierro no hemo. Estas pueden acelerar la oxidación de los lípidos generando el oxígeno activo durante la autooxidación de la $\mathrm{Hb}$ (Schricker y Miller, 1983), pues a la muerte del pez, el átomo de hierro del anillo hemo de las hemoproteínas se encuentra en estado ferroso $(+2)$ y durante la autooxidación es convertido a meta $(+3)$ hemoproteína (meta-HP). En la autooxidación, la meta-HP reacciona con los peróxidos para formar 


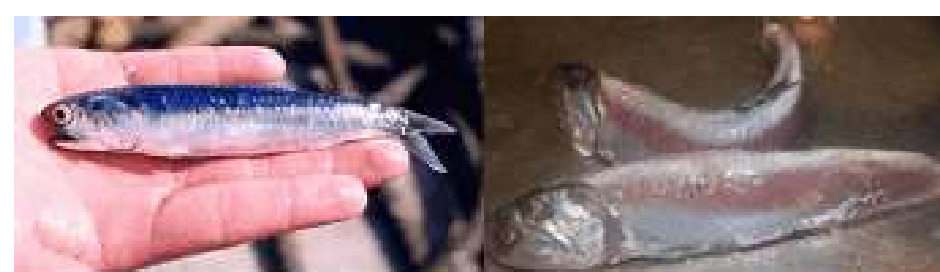

(a) Anchoveta entera (b) Desollado entero

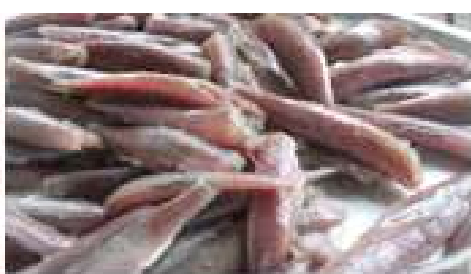

(c) Descabezado y eviscerado

Figura 1.Anchoveta entera, desollada, descabezada y eviscerada previa a su congelación

compuestos capaces de iniciar y propagar la oxidación de lípidos. Es por esto que se han utilizado agentes quelantes para retener los iones libres o antioxidantes que suministren electrones apareados a los iones libres (Ahn et al., 1993; Salas et al., 2007; Taheri et al., 2012). Por ejemplo, el citrato sódico en filetes de salmón «Onchorhynchus nerka» contribuyó, debido a su efecto quelante, en la prevención de la oxidación de grasa en comparación con el uso de otras sales orgánicas como el lactato sódico y acetato sódico (Ibrahim, 2007).

La substitución del uso de sal común (cloruro sódico) por el citrato sódico en la etapa del desangrado de la anchoveta podría evitar la oxidación. El citrato sódico es una de las sales más prometedoras por sus diversas características, tales como la quelación de metales (mayor constante de quelación ante hierro y calcio) y la posesión de varios grupos ionizables (tres grupos carboxilos). Además, el citrato sódico, al ser un osmolito inactivo, tiene un efecto anticoagulante de la sangre debido a la quelación de iones y protección del glóbulo rojo, lo cual evita la formación de más hierro libre (Salvay et al., 2003).

El objetivo del estudio fue evaluar el efecto del desollado y desangrado de anchoveta (Engraulis ringens) en solución de citrato sódico a fin de determinar su periodo de vida útil congelada en almacenamiento a $-25{ }^{\circ} \mathrm{C}$.

\section{Materiales Y MéTodos}

\section{Materia Prima}

La producción primaria de la anchoveta se realizó frente a la costa del Puerto de San Andrés, Pisco (Ica, Perú), a una latitud de $13^{\circ} 42^{\prime} 55^{\prime}$ 'S. La anchoveta entera $(90 \mathrm{~kg})$ fue recibida a $2{ }^{\circ} \mathrm{C}$, proveniente de una sola cala, e inmediatamente transportada a las instalaciones de la empresa pesquera Pezex S.A. (Pisco, Ica) para su recepción y preparación.

\section{Preparación de la Muestra}

Luego de $5 \mathrm{~h}$ de captura, la anchoveta entera (Fig. 1a) fue desollada (Fig. 1b) a través de su inmersión y agitación fuerte en agua caliente $\left(85^{\circ} \mathrm{C}\right.$ por $\left.15 \mathrm{~s}\right)$, lavada con agua a alta presión e inmediatamente enfriada en agua $\left(2{ }^{\circ} \mathrm{C}\right)$. Posteriormente, se realizó el descabezado y eviscerado (Fig. 1c), obteniendo $36 \mathrm{~kg}$ de anchoveta desollada, descabezada y eviscerada (ADDE). Previo al desangrado, los ejemplares fueron separados en dos grupos: $15 \mathrm{~kg}$ ADDE (Control) y $21 \mathrm{~kg}$ ADDE (Tratamiento). El desangrado consistió en sumergir el grupo Control en agua y el grupo Tratamiento en una solución de citrato sódico $(2.5 \%)$ en una proporción 2:1 (diluyente:ADDE), ambos a $2{ }^{\circ} \mathrm{C}$ durante $1 \mathrm{~h}$.

Los ejemplares de ADDE fueron estibados en una caja de capacidad de $2 \mathrm{~kg}$ y posteriormente (10 h luego de su captura) 
Cuadro 1. Descriptores y valores usados en la evaluación sensorial de anchoveta desollada, descabezada y eviscerada

\begin{tabular}{cll}
\hline Puntuación & Calificación & Descriptores de olor y sabor \\
\hline 5 & Muy Bueno & $\begin{array}{l}\text { Olor y sabor muy agradable a pescado fresco } \\
\text { característico de anchoveta cocida }\end{array}$ \\
4 & Bueno & Olor y sabor moderado a pescado \\
3 & Aceptable & Olor y sabor intenso a pescado con ligera rancidez \\
2 & Malo & Olor y sabor con moderada rancidez, desagradable \\
1 & Muy malo & Olor y sabor con intensa rancidez \\
\hline
\end{tabular}

congelados por aire forzado a $-30{ }^{\circ} \mathrm{C}$ durante $8 \mathrm{~h}$. El producto congelado fue colocado dentro de una caja isotérmica con trozos de hielo seco $\left(\mathrm{CO}_{2}\right.$ sólido) y transportado al Instituto Tecnológico de la Producción (ITP) (Callao, Perú). A su llegada al ITP, las muestras congeladas Control y Tratamiento fueron glaseadas por inmersión en agua y en solución de citrato sódico $(2.5 \%)$, respectivamente, a $2{ }^{\circ} \mathrm{C}$ por $10 \mathrm{~s}$. Después del glaseado, las muestras fueron colocadas nuevamente en sus cajas y almacenadas a $-25^{\circ} \mathrm{C}$.

\section{Acondicionamiento}

Previo a los análisis, las muestras congeladas fueron colocadas en refrigeración $\left(2{ }^{\circ} \mathrm{C}\right)$ durante $12 \mathrm{~h}$ hasta alcanzar aproximadamente $0.4{ }^{\circ} \mathrm{C}$ en el centro de la muestra. El remanente de glaseado fue retirado con papel adsorbente de humedad.

Los análisis fueron realizados mensualmente en el Laboratorio Fisicoquímico-Sensorial del ITP. Los análisis químicos y sensoriales fueron realizados por un periodo de 10 y 14 meses de almacenamiento, respectivamente.

\section{Análisis Químico}

Todos los reactivos utilizados fueron de grado químico. Los análisis fueron realizados por duplicado.
- La composición proximal fue realizada de acuerdo a la metodología recomendada por FAO (1996).

- La determinación de hierro hemínico fue analizado en el músculo oscuro, músculo claro y músculo total de acuerdo a lo señalado por Thiansilakul et al. (2010).

- Las sustancias reactivas al ácido tiobarbitúrico (SRAT) se determinaron según Vyncke (1975), expresadas como mg malonaldehído/kg muestra.

- $\quad$ El pH fue determinado en una mezcla $(10 \%)$ de muestra triturada diluida con agua desionizada y leído directamente por un electrodo (Sensorex) acoplado a un potenciómetro (Mettler Toledo, modelo S20K Seven Easy, EEUU).

- El análisis del valor peróxido (VP) fue expresado en meq oxígeno peroxídico (AOAC: 965.33) y la acidez (A) expresada como porcentaje de ácido oleico (AOAC: 940.28).

- El perfil de ácidos grasos (PAG) por cromatografía de gases (Prevot y Mordret, 1976), se realizó en la grasa extraída de la muestra de acuerdo al método de Bligh y Dyer (1959).

\section{Análisis Sensorial}

Seis ejemplares de anchoveta de cada grupo fueron colocadas en bolsas de polietileno/nylon y cocinados por vapor de agua durante $5 \mathrm{~min}$. Luego se hizo la evalua- 
Cuadro 2. Composición proximal de anchoveta ${ }^{1}$

\begin{tabular}{lccc}
\hline Componente & $\begin{array}{c}\text { Anchoveta entera } \\
\text { con piel }\end{array}$ & $\begin{array}{c}\text { Anchoveta DDE } \\
(\text { Control })\end{array}$ & $\begin{array}{c}\text { Anchoveta DDE } \\
\text { (Tratamiento) }\end{array}$ \\
\hline Humedad (\%) & $75.2^{\mathrm{a}} \pm 0.4$ & $78.2^{\mathrm{b}} \pm 0.1$ & $80.5^{\mathrm{c}} \pm 0.3$ \\
Grasa (\%) & $3.4^{\mathrm{a}} \pm 0.1$ & $1.2^{\mathrm{b}} \pm 0.3$ & $1.4^{\mathrm{b}} \pm 0.2$ \\
Proteína (\%) & $19.8^{\mathrm{a}} \pm 0.5$ & $19.7^{\mathrm{a}} \pm 0.5$ & $16.7^{\mathrm{b}} \pm 0.3$ \\
Ceniza (\%) & $1.8^{\mathrm{a}} \pm 0.0$ & $1.1^{\mathrm{b}} \pm 0.15$ & $1.8^{\mathrm{a}} \pm 0.0$ \\
\hline${ }^{1}$ Los valores de composición proximal corresponden al promedio de dos repeticiones \\
${ }^{\mathrm{a}, \mathrm{b}, \mathrm{c}}$ Superíndices distintos dentro de filas indican diferencia significativa entre muestras $(\mathrm{p}<0.05)$
\end{tabular}

ción de los atributos de olor y sabor por seis panelistas entrenados, según los descriptores del Cuadro 1 (Maza et al., 2008). Las muestras fueron calificadas como mala o rechazada cuando la puntuación alcanzaba el valor de 2 (escala de 1 a 5 ).

\section{Análisis Estadístico}

Se utilizó el programa estadístico IBM SPSS Statistics 18 en el análisis de varianza (ANOVA) y la prueba de Tukey para las comparaciones múltiples a un nivel de significancia de 0.05 para los análisis químicos, y la prueba no paramétrica de KruskalWallis para la evaluación sensorial.

\section{Resultados y Discusión}

\section{Composición Proximal}

Los ejemplares de anchoveta entera tuvieron un peso promedio de $19.0 \pm 0.9 \mathrm{~g} \mathrm{y}$ longitud total de $14.3 \pm 0.9 \mathrm{~cm}$, cumpliendo con la talla mínima de captura indicada por el IMARPE (2013). Sus valores de humedad, grasa, proteína $\left(\% \mathrm{~N}^{*} 6.25\right)$ y ceniza se muestran en el Cuadro 2.

Según FAO (1999), los pescados grasos contienen de 8 a $15 \%$ de grasa (caballa, sardina, arenque, atún); no obstante, la anchoveta es considerada como un pescado graso con registro de valores de grasa variable de $3.9 \%$
(Salas et al., 2007), 10.7\% (Maza et al., 2008) hasta 11.6\% (Karacam y Boran, 1996). Sin embargo, en el presente estudio los filetes con piel de la anchoveta entera presentaron un bajo contenido de grasa (3.4\%), donde el desollado retiró el 59 y $65 \%$ del contenido inicial de grasa, resultando filetes con 1.4 y $1.2 \%$ de grasa para la muestras Control y Tratamiento, respectivamente (Cuadro 2). Estos podrían considerarse como filetes magros menores de $2 \%$ de grasa (FAO, 1999). Similar resultado fue encontrado por Salas et al. (2004) en jurel «Trachurus murphyi», donde el desollado retiró el $66.8 \%$ de grasa a partir de los filetes con piel $(6.6 \%$ grasa) para obtener la pulpa de pescado (2.2\% grasa). El desollado retira una importante cantidad de grasa subcutánea proveniente de la piel del pescado y, por consiguiente, se obtiene un producto con menor contenido graso y podría presentar menor susceptibilidad a la oxidación lipídica.

La relación del músculo claro y músculo oscuro en el filete de anchoveta fue alrededor de 4:1 (claro: oscuro) (datos no mostrados).

\section{Análisis Químicos}

Las figuras 1,2 y 3 muestran los resultados del análisis del deterioro de la grasa (valor peróxido, acidez y sustancias reactivas al ácido barbitúrico) en las muestras de anchoveta. 


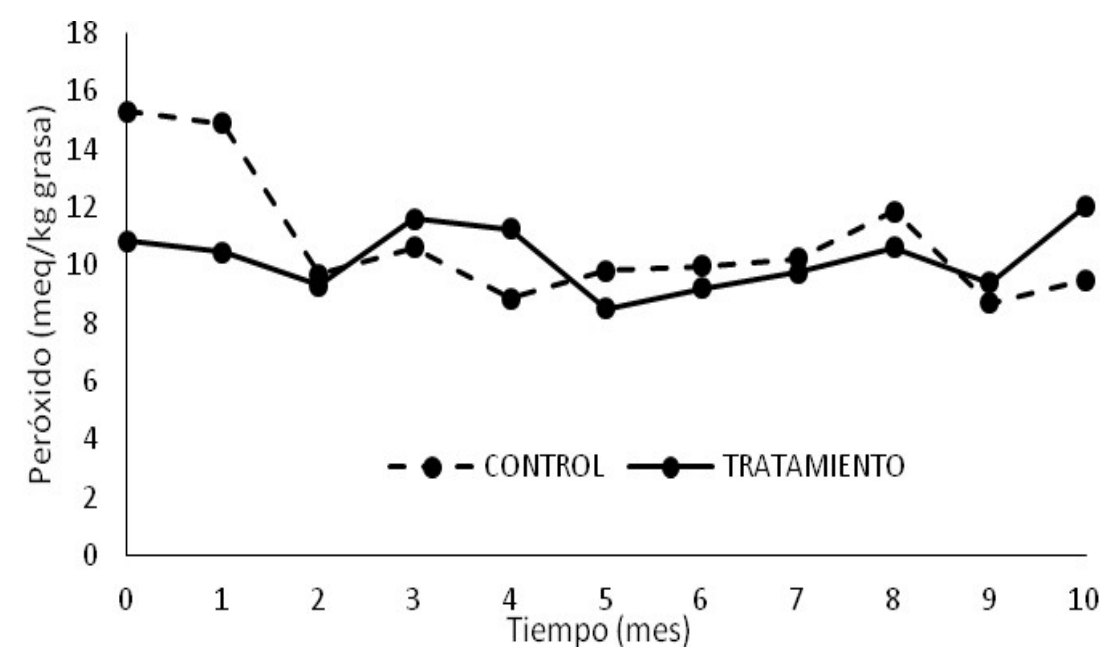

Figura 1. Comportamiento del valor peróxido (VP) de ejemplares de anchoveta desollada, descabezada y eviscerada (ADDE) almacenada a $-25{ }^{\circ} \mathrm{C}$ durante 10 meses

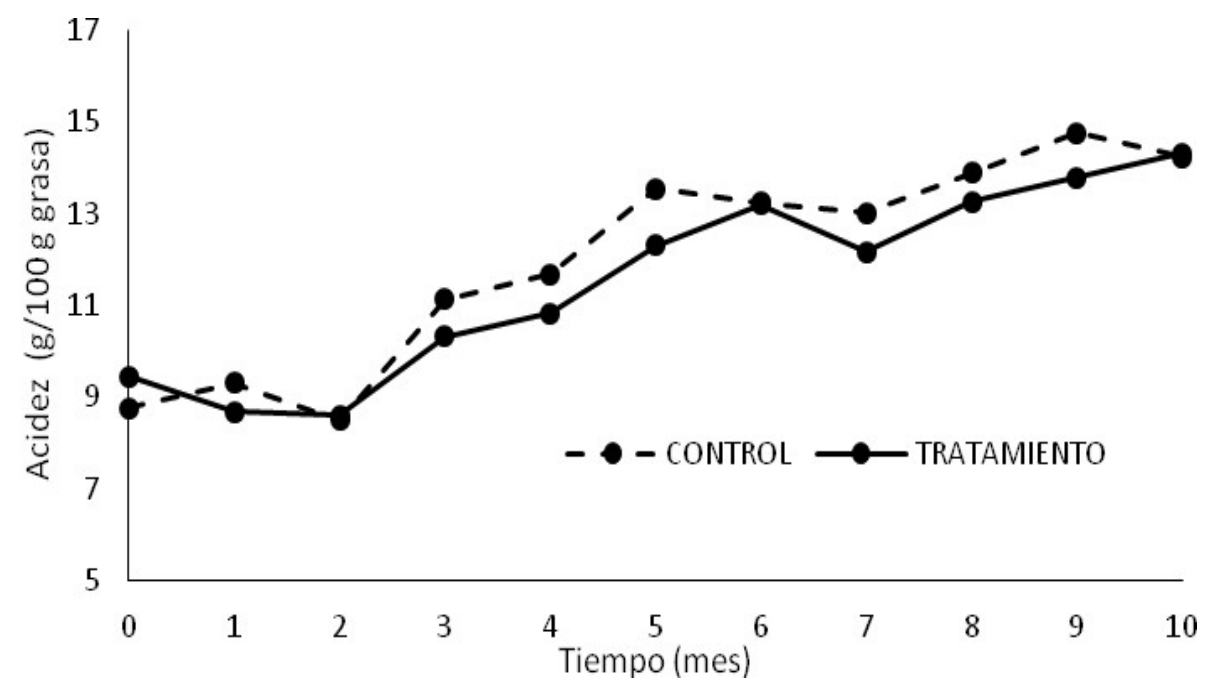

Figura 2. Comportamiento de la acidez (A) de anchoveta desollada, descabezada y eviscerada (ADDE) almacenada a $-25{ }^{\circ} \mathrm{C}$ durante 10 meses

Los valores del peróxido presentaron diferencias significativas $(\mathrm{p}<0.05)$, siendo inicialmente de $15.3 \mathrm{meq} / \mathrm{kg}$ y $10.8 \mathrm{meq} / \mathrm{kg}$ para el Control y Tratamiento, respectivamente, llegando a disminuir y mostrando fluctuaciones con valores promedio de $9.9 \mathrm{meq} / \mathrm{kg}$ para Control y $10.2 \mathrm{meq} / \mathrm{kg}$ para Tratamiento. Los valores iniciales fueron similares a los encontrados por Salas et al. (2004) con 8.0 $\mathrm{meq} / \mathrm{kg}$ en pulpa de jurel «Trachurus murphyi».
Los valores de acidez (Figura 2) y SRAT (Figura 3 ) presentaron diferencias significativas $(\mathrm{p}<0.05)$ durante los 10 meses de almacenamiento, observándose un incremento significativo en el tiempo $(p<0.05)$. Todos los resultados de análisis del deterioro de grasa para el Control (VP: $15.30 \mathrm{meq} / \mathrm{kg}$; A: 8.76\% y SRAT: $8.04 \mathrm{mg} / \mathrm{kg}$ ) y el Tratamiento (VP: $10.85 \mathrm{meq} / \mathrm{kg}$; A: $9.46 \%$ y SRAT: $3.48 \mathrm{mg} / \mathrm{kg}$ ) en el inicio del estudio fueron significativamente diferentes $(\mathrm{p}<0.05)$ y mayores (de 


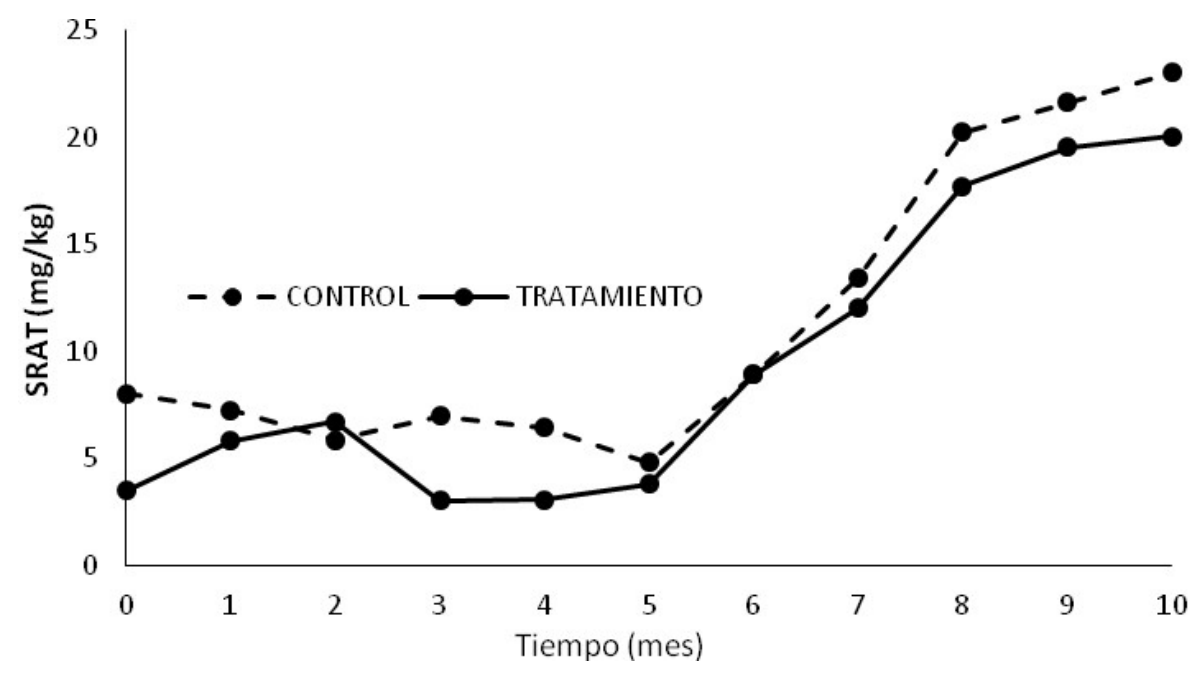

Figura 3. Comportamiento de las sustancias reactivas al ácido tiobarbitúrico (SRAT) en anchoveta desollada, descabezada y eviscerada (ADDE) almacenada a $-25^{\circ} \mathrm{C}$ durante 10 meses

3 a 8 veces más) a los valores obtenidos por Rezaei y Hosseini (2008) (VP: $1.2 \mathrm{meq} / \mathrm{kg}$; A: $1.5 \%$; SRAT: $0.05 \mathrm{mg} / \mathrm{kg})$, Karacam y Boran (1996) (VP: $1.8 \mathrm{meq} / \mathrm{kg}$; A: 2.0\%; SRAT: $0.3 \mathrm{mg} / \mathrm{kg}$ ), Aubourg y Ugliano (2002) (VP: $1.5 \mathrm{meq} / \mathrm{kg}$; A: $1.15 \%$; SRAT: $0.12 \mathrm{mg} /$ $\mathrm{kg}$ ), Maza et al. (2008) (VP: $5.0 \mathrm{meq} / \mathrm{kg} ; \mathrm{A}$ : $0.12 \%$; SRAT: $0.60 \mathrm{mg} / \mathrm{kg}$ ) y Salas et al. (2004) (VP: $8.0 \mathrm{meq} / \mathrm{kg}$; SRAT: $1.18 \mathrm{mg} / \mathrm{kg}$ ).

Los altos resultados registrados en el presente estudio no indican que la anchoveta «Engraulis ringens» se encontrase en deterioro o rechazada sensorialmente, como lo sugiere Kurade y Baranowski (1987, citado por Salas et al., 2007), donde indicó que un pescado congelado almacenado es aceptable sensorialmente con un contenido máximo de SRAT de $4 \mathrm{mg} / \mathrm{kg}$. En este estudio, los valores iniciales de SRAT fueron mayores de $4 \mathrm{mg} / \mathrm{kg}$ en ambos grupos, y estos valores fluctuaron hasta el quinto mes, donde se incrementaron gradualmente hasta los 10 meses de almacenamiento, permaneciendo sensorialmente aceptables (Figura 3 ).

Los análisis de valor peróxido (VP) y acidez (A) son reportados en función de la cantidad de grasa de la muestra. Si estas tuvieran una cantidad de grasa de 5,8 o 10 , se reflejarían los compuestos de deterioro en los análisis de VP y A, siendo rechazados sensorialmente; pero si las muestras tuvieran una mínima cantidad de grasa como en el presente estudio (1.4\% para el Control y $1.2 \%$ para el Tratamiento), estas muestras serían aceptables sensorialmente aun si presentasen altos valores de VP y A, respecto a los valores predeterminados. Por ello, se propone como el «efecto de concentración» a la cantidad mínima de grasa que concentraría altos valores de VP y A que no son percibidos ni rechazados sensorialmente. Esto, a diferencia de otras muestras en pescados con mayores contenidos de grasa, como $3.9 \%$ (Salas et al., 2007), 10.7\% (Maza et al., 2008) y hasta $11.6 \%$ (Karacam y Boran, 1996), donde los compuestos de oxidación fueron fácilmente perceptibles e influyeron en el corto periodo de vida útil de las muestras evaluadas (4 a 6 meses) en comparación con los 14 meses determinados en el presente estudio.

Los valores de $\mathrm{pH}$ de las muestras no presentaron diferencias significativas durante el almacenamiento (datos no mostrados). La muestra Control presentó un $\mathrm{pH}$ alrededor de $6.3 \mathrm{y}$ el Tratamiento de 6.7. El valor de $\mathrm{pH}$ del tratamiento fue debido a su inmersión en la solución de citrato (2.5\%) durante 
Cuadro 3. Hierro hemínico $(\mu \mathrm{g} / \mathrm{g})$ en las muestras de anchoveta desollada, descabezada y eviscerada $\left(\mathrm{ADDE}^{1}\right)$ almacenadas a $-25^{\circ} \mathrm{C}$ por 10 meses

\begin{tabular}{ccccccc}
\hline \multirow{2}{*}{ Mes } & \multicolumn{3}{c}{ Control } & \multicolumn{3}{c}{ Tratamiento } \\
\cline { 2 - 7 } & Total & Oscuro & Claro & Total & Oscuro & Claro \\
\hline 0 & $7.0^{\mathrm{a}} \pm 0.3$ & $27.0^{\mathrm{b}} \pm 0.8$ & $4.3^{\mathrm{a}} \pm 0.2$ & $9.5^{\mathrm{b}} \pm 0.5$ & $26.9^{\mathrm{b}} \pm 0.4$ & $4.0^{\mathrm{a}} \pm 0.6$ \\
1 & $8.4^{\mathrm{b}} \pm 0.1$ & $27.4^{\mathrm{b}} \pm 0.7$ & $6.3^{\mathrm{d}} \pm 0.4$ & $8.9^{\mathrm{a}} \pm 0.2$ & $22.9^{\mathrm{a}} \pm 0.6$ & $6.4^{\mathrm{b}} \pm 0.4$ \\
2 & $10.3^{\mathrm{c}} \pm 0.2$ & $24.5^{\mathrm{a}} \pm 0.4$ & $5.7^{\mathrm{c}} \pm 0.4$ & $8.9^{\mathrm{a}} \pm 0.1$ & $28.4^{\mathrm{d}} \pm 0.6$ & $4.8^{\mathrm{a}} \pm 0.0$ \\
3 & $6.1^{\mathrm{a}} \pm 0.5$ & $21.4^{\mathrm{a}} \pm 1.4$ & $5.8^{\mathrm{c}} \pm 0.2$ & $7.5^{\mathrm{a}} \pm 0.3$ & $25.4^{\mathrm{a}} \pm 0.8$ & $5.1^{\mathrm{a}} \pm 0.4$ \\
4 & $8.3^{\mathrm{b}} \pm 0.1$ & $31.1^{\mathrm{d}} \pm 0.1$ & $5.7^{\mathrm{b}} \pm 0.2$ & $8.0^{\mathrm{a}} \pm 0.9$ & $30.7^{\mathrm{e}} \pm 1.8$ & $5.2^{\mathrm{a}} \pm 0.5$ \\
5 & $7.6^{\mathrm{a}} \pm 0.5$ & $30.8^{\mathrm{c}} \pm 1.4$ & $5.2^{\mathrm{a}} \pm 0.1$ & $9.6^{\mathrm{b}} \pm 0.2$ & $24.4^{\mathrm{a}} \pm 0.4$ & $5.4^{\mathrm{a}} \pm 0.2$ \\
6 & $10.3^{\mathrm{c}} \pm 0.9$ & $33.1^{\mathrm{e}} \pm 1.1$ & $5.5^{\mathrm{b}} \pm 0.9$ & $11.9^{\mathrm{c}} \pm 0.2$ & $27.4^{\mathrm{b}} \pm 0.6$ & $7.4^{\mathrm{c}} \pm 1.0$ \\
7 & $7.6^{\mathrm{a}} \pm 0.0$ & $30.4^{\mathrm{c}} \pm 1.2$ & $4.8^{\mathrm{a}} \pm 0.1$ & $7.5^{\mathrm{a}} \pm 0.0$ & $25.0^{\mathrm{a}} \pm 0.4$ & $5.6^{\mathrm{a}} \pm 0.8$ \\
8 & $7.8^{\mathrm{a}} \pm 0.2$ & $26.2^{\mathrm{b}} \pm 0.7$ & $4.0^{\mathrm{a}} \pm 0.1$ & $8.2^{\mathrm{a}} \pm 0.3$ & $27.5^{\mathrm{c}} \pm 1.1$ & $5.8^{\mathrm{a}} \pm 0.4$ \\
9 & $7.4^{\mathrm{a}} \pm 0.8$ & $25.8^{\mathrm{b}} \pm 1.8$ & $4.9^{\mathrm{a}} \pm 0.1$ & $7.4^{\mathrm{a}} \pm 0.1$ & $25.8^{\mathrm{a}} \pm 0.5$ & $4.2^{\mathrm{a}} \pm 0.2$ \\
10 & $8.2^{\mathrm{b}} \pm 0.5$ & $24.3^{\mathrm{a}} \pm 0.7$ & $5.3^{\mathrm{a}} \pm 0.2$ & $8.5^{\mathrm{a}} \pm 0.8$ & $25.9^{\mathrm{a}} \pm 0.5$ & $5.2^{\mathrm{a}} \pm 0.1$ \\
\hline
\end{tabular}

${ }^{1}$ Los resultados son el promedio de dos repeticiones

$a, b, c, d$ Letras con superíndices distintas dentro de columnas indican diferencia significativa $(p<0.05)$

el desangrado, cuya constante de ionización $(\mathrm{pka}=8.3)$ permitió elevar el $\mathrm{pH}$ en 0.4 unidades respecto al control.

Los valores del hierro hemínico en el músculo total, claro y oscuro de las muestras control y tratamiento se muestran en el Cuadro 3. Existe diferencia significativa $(\mathrm{p}<0.05)$ en la concentración de hierro hemínico entre el músculo claro y músculo oscuro, teniendo este último una concentración cinco veces mayor. Asimismo, Chaijan et al. (2005) encontró entre 2 y 4 veces mayor contenido de hierro hemínico en el músculo oscuro que en el músculo claro en filetes de sardina «Sardinella gibbosa» y caballa «Rastrelliger kanagurta» durante 15 días de almacenamiento a $4{ }^{\circ} \mathrm{C}$. La mayor concentración de hierro hemínico refleja el mayor contenido de mioglobina y coloración rojiza del músculo oscuro respecto al músculo claro; además, el hierro hemínico representa entre el 25 al 44\% del total de hierro en pescados (Fisher y Deng, 1977, citado por Chaijan et al., 2005). Esto es importante pues la mayor asimilación del hierro por el organismo es en estado de hierro hemínico (estado ferroso [+2] que forma parte del grupo hemo de la mioglobina y hemoglobina) y no en estado de hierro no hemo o en estado férrico $(+3)$, que por el contrario acelera el proceso de deterioro oxidando la grasa (Ahn et al., 1993). Es por esto que, según el Cuadro 3, el contenido de hierro hemínico tuvo una tendencia fluctuante pero no un descenso marcado; es decir, la metodología experimental permitió la conservación del hierro en estado hemínico que no propició un rápido deterioro de las muestras estudiadas.

Otros autores han registrado resultados de hierro hemínico, como $5.6 \mu \mathrm{g} / \mathrm{g}$ en anchoveta «Engraulis encrasicholus» (Turhan et al., 2004), $6.9 \mu \mathrm{g} / \mathrm{g}$ en músculo de pescado lubina «Lates calcarifer»; $5.6 \mu \mathrm{g} / \mathrm{g}$ en músculo de tilapia roja «Oreochromis mossambicus $x$ O. niloticus» (Thiansilakul et al., 2010) y $53 \mu \mathrm{g} / \mathrm{g}$ en la trucha arcoíris «Oncorhynchus mykiss» (Rezaei y Hosseini, 
Cuadro 4. Composición de ácidos grasos (\%) en la muestra Control de anchoveta

\begin{tabular}{lcccccc}
\hline \multirow{2}{*}{ Ácidos grasos } & \multicolumn{5}{c}{ Tiempo de almacenamiento (meses) } \\
\cline { 2 - 7 } & 0 & 2 & 4 & 6 & 8 & 10 \\
\hline C14:0 (Ac. mirístico) & 4.82 & 5.14 & 5.07 & 5.33 & 5.18 & 5.61 \\
C15:0 (Ac. pentadecanoico) & 0.35 & 0.36 & 0.38 & 0.39 & 0.38 & 0.38 \\
C16:0 (Ac. palmítico) & 21.71 & 20.95 & 23.38 & 22.84 & 23.14 & 23.43 \\
C17:0 (Ac. heptadecanoico) & 0.34 & 0.33 & 0.35 & 0.37 & 0.36 & 0.34 \\
C18:0 (Ac. esteárico) & 3.78 & 3.81 & 3.97 & 4.04 & 3.97 & 4.10 \\
C20:0 (Ac. araquídico) & 0.26 & 0.15 & 0.22 & 0.17 & 0.14 & 0.15 \\
\hline Tota1 saturados & 31.26 & 30.74 & 33.37 & 33.14 & 33.17 & 34.01 \\
\hline C16:1 (Ac. palmitoleico) & 4.69 & 4.56 & 5.04 & 5.17 & 5.15 & 5.36 \\
C18:1 (Ac. oleico) w9 & 6.83 & 6.75 & 6.94 & 6.61 & 6.93 & 8.10 \\
C18:1 (Ac. vaccénico) w7 & 2.67 & 2.58 & 2.72 & 2.59 & 2.52 & 2.59 \\
C20:1 (Ac. eicosaenoico) & 0.24 & 0.26 & 0.19 & 0.23 & 0.24 & 0.23 \\
\hline Total monoinsaturados & 14.43 & 14.15 & 14.89 & 14.60 & 14.84 & 16.28 \\
\hline C18:3 (Ac. $\alpha$ linolénico) w3 & 0.50 & 0.34 & 0.36 & 0.40 & 0.33 & 0.36 \\
C18:4 (Ac. estearidónico) w3 & 1.15 & 1.35 & 1.24 & 1.27 & 1.27 & 1.38 \\
C20:3 (Ac. eicosatrienoico) w3 & 1.13 & 1.07 & 1.02 & 1.06 & 1.07 & 1.05 \\
C20:5 (Ac. eicosapentanoico) w3 & 14.63 & 13.55 & 13.60 & 13.85 & 13.86 & 14.28 \\
C22:5 (Ac. clupadónico) w3 & 2.54 & 2.52 & 2.29 & 2.33 & 2.25 & 2.32 \\
C22:6 (Ac. docosahexaenoico) w3 & 31.79 & 31.27 & 30.58 & 29.94 & 29.36 & 27.57 \\
C18:2 (Ac. linoleico) w6 & 0.8 & 0.76 & 0.80 & 0.78 & 0.74 & 0.71 \\
\hline Total poliinsaturados & 52.54 & 50.86 & 49.89 & 49.63 & 48.88 & 47.67 \\
\hline Total ácidos grasos & 98.23 & 95.75 & 98.15 & 97.37 & 96.86 & 97.96 \\
\hline
\end{tabular}

2008). Dichos valores indican que existe una amplia variabilidad de hierro hemínico en pescados, probablemente por sus diferentes proporciones de músculo oscuro, alimentación, etc. Rezaei y Hosseini (2008) reportaron una disminución significativa de hierro hemínico en la trucha arcoíris sumergida en hielo durante 20 días de almacenamiento que fue rechazada sensorialmente a los 12 días, lo cual indica que el contenido de hierro hemo disminuye mientras que el contenido de hierro no hemo aumenta conforme el pescado se va deteriorando, el cual cataliza la oxidación de lípidos desarrollando un mal sabor y olor a pescado intenso. Esto fue, asimismo, reportado por Maqsood y Benjakul (2011). Esto indicaría en el presente estudio que los compuestos de hierro hemínico, principalmente mioglobina, presentes en el músculo de la anchoveta fueron estables y no fueron liberados como hierro no hemo, pues el hierro hemínico no presentó un descenso constante durante el periodo de almacenamiento (Cuadro 3 ). 
Cuadro 5. Composición de ácidos grasos (\%) en la muestra Tratamiento de anchoveta

\begin{tabular}{lcccccc}
\hline \multirow{2}{*}{ Ácidos grasos } & \multicolumn{6}{c}{ Tiempo de almacenamiento (meses) } \\
\cline { 2 - 7 } & 0 & 2 & 4 & 6 & 8 & 10 \\
\hline C14:0 (Ac. mirístico) & 5.05 & 4.99 & 5.72 & 5.72 & 5.39 & 4.85 \\
C15:0 (Ac. pentadecanoico) & 0.34 & 0.37 & 0.4 & 0.38 & 0.40 & 0.36 \\
C16:0 (Ac. palmítico) & 22.7 & 21.41 & 24.81 & 23.35 & 23.00 & 21.43 \\
C17:0 (Ac. heptadecanoico) & 0.32 & 0.36 & 0.48 & 0.37 & 0.38 & 0.34 \\
C18:0 (Ac. esteárico) & 3.89 & 4.17 & 0.13 & 4.37 & 4.48 & 4.04 \\
C20:0 (Ac. araquídico) & 0.18 & 0.18 & 0.20 & 0.15 & 0.15 & 0.16 \\
\hline Total saturados & 32.48 & 31.48 & 31.74 & 34.34 & 33.80 & 31.18 \\
\hline C16:1 (Ac. palmitoleico) & 4.57 & 4.93 & 5.30 & 5.41 & 5.28 & 4.97 \\
C18:1 (Ac. oleico) w9 & 7.12 & 6.34 & 7.17 & 7.49 & 7.49 & 7.06 \\
C18:1 (Ac. vaccénico) w7 & 2.59 & 2.64 & 2.53 & 2.66 & 2.85 & 2.59 \\
C20:1 (Ac. eicosaenoico) & 0.20 & 0.26 & 0.17 & 0.24 & 0.23 & 0.25 \\
\hline Total monoinsaturados & 14.48 & 14.17 & 15.17 & 15.80 & 15.85 & 14.87 \\
\hline C18:3 (Ac. $\alpha$ linolénico) w3 & 0.3 & 0.33 & 0.40 & 0.34 & 0.34 & 0.34 \\
C18:4 (Ac. estearidónico) w3 & 1.04 & 1.34 & 1.26 & 1.18 & 1.40 & 1.31 \\
C20:3 (Ac. eicosatrienoico) w3 & 1.01 & 1.10 & 1.01 & 0.92 & 1.09 & 1.08 \\
C20:5 (Ac. eicosapentanoico) w3 & 14.69 & 14.49 & 13.75 & 13.57 & 13.43 & 14.76 \\
C22:5 (Ac. clupadónico) w3 & 2.65 & 2.37 & 2.11 & 2.29 & 2.21 & 3.03 \\
C22:6 (Ac. docosahexaenoico) w3 & 30.67 & 30.90 & 27.94 & 28.04 & 26.94 & 30.2 \\
C18:2 (Ac. linoleico) w6 & 0.96 & 0.75 & 0.74 & 0.75 & 0.79 & 0.78 \\
\hline Total poliinsaturados & 51.32 & 51.28 & 47.21 & 47.09 & 46.2 & 51.5 \\
\hline Total ácidos grasos & 98.28 & 96.93 & 94.12 & 97.23 & 95.85 & 97.55 \\
\hline
\end{tabular}

Cuadro 6. Evaluación sensorial (escala de 1 a 5$)^{1}$ de las muestras de anchoveta almacenadas por 14 meses de $-25^{\circ} \mathrm{C}$

\begin{tabular}{lcccccccccccccc}
\hline & \multicolumn{11}{c}{ Meses de almac enamiento } \\
\cline { 2 - 5 } & 0 & 1 & 2 & 3 & 4 & 5 & 6 & 7 & 8 & 9 & 10 & 11 & 12 & 14 \\
\hline Control & $5^{\mathrm{a}}$ & $5^{\mathrm{a}}$ & $5^{\mathrm{a}}$ & $5^{\mathrm{a}}$ & $5^{\mathrm{a}}$ & $4.8^{\mathrm{a}}$ & $4.8^{\mathrm{a}}$ & $4.8^{\mathrm{a}}$ & $4.6^{\mathrm{b}}$ & $4.6^{\mathrm{b}}$ & $4.4^{\mathrm{b}}$ & $4.0^{\mathrm{c}}$ & $3.9^{\mathrm{c}}$ & $3.6^{\mathrm{d}}$ \\
Tratamiento & $5^{\mathrm{a}}$ & $5^{\mathrm{a}}$ & $5^{\mathrm{a}}$ & $5^{\mathrm{a}}$ & $5^{\mathrm{a}}$ & $5^{\mathrm{a}}$ & $5^{\mathrm{a}}$ & $4.8^{\mathrm{b}}$ & $4.8^{\mathrm{b}}$ & $4.6^{\mathrm{b}}$ & $4.6^{\mathrm{b}}$ & $4.5^{\mathrm{b}}$ & $4.2^{\mathrm{c}}$ & $3.9^{\mathrm{d}}$ \\
\hline
\end{tabular}

Los resultados corresponden al promedio de seis panelistas entrenados. Muy bueno (5), Bueno (4), Aceptable (3), Malo (2), Muy malo (1)

${ }_{a, b, c, d}$ Valores promedio en la misma fila con diferente superíndice son significativamente diferentes $(p<0.05)$ 
Según los cuadros 4 y 5, los tres ácidos grasos mayoritarios en la carne de anchoveta del presente estudio fueron el ácido docosahexaenoico (C22:6), ácido palmítico (C16:0) y ácido eicosapentanoico (C20:5). Asimismo, el ácido graso palmítico fue el más abundante $(69.2 \%$ en el Control y $70.1 \%$ en el Tratamiento) respecto a los ácidos grasos saturados, y los ácidos grasos docosahexaenoico (DHA) $(60.3 \%$ en el Control y $59.3 \%$ en el Tratamiento) y eicosapentaenoico (EPA) $(28.0 \%$ en el Control y $28.7 \%$ en el Tratamiento) fueron los mayoritarios respecto a los ácidos grasos poliinsaturados.

Estos resultados fueron similares a los reportados por González et al. (2004) y Albrecht y Salas (2015). No obstante, las cantidades de ácidos grasos de ambas muestras estudiadas presentaron diferencias significativas $(\mathrm{p}<0.05)$, siendo mayor los ácidos grasos poliinsaturados (AGP), seguidos de los ácidos grasos saturados (AGS) y de los ácidos grasos monoinsaturados (AGM). Este orden podría ser útil para identificar a los pescados, respecto a la carne de los animales terrestres, considerando que para la carne de vacuno, pollo, alce y búfalo se registra una mayor cantidad de AGS seguido de los AGP (Rule et al., 2002).

De acuerdo a las variaciones de resultados de los ácidos grasos poliinsturados (Cuadros 4 y 5), las muestras de ambos grupos presentaron una disminución máxima de alrededor de $10 \%$ en su contenido, sin embargo, otros estudios reportaron alrededor del 11\% (González et al., 2004) y hasta 34\% (Memon et al., 2010). Esto indica que los ácidos grasos poliinsaturados del presente estudio presentaron estabilidad durante el periodo de almacenamiento a $-25^{\circ} \mathrm{C}$ que contribuiría en su aceptabilidad sensorial (Cuadro 6).

\section{Análisis Sensorial}

Las muestras de los grupos Control y Tratamiento fueron aceptables hasta los 14 meses de almacenamiento a $-25{ }^{\circ} \mathrm{C}$ (Cuadro
6), habiendo diferencias significativas $(p<0.05)$ entre los valores promedio de aceptación de las muestras durante el periodo de estudio.

Karacam y Boran (1996), Salas et al. (2004), Salas et al.(2007), Maza et al. (2008), Taheri et al. (2012) determinaron un periodo de alrededor de 6 meses de vida útil en distintos pescados (anchoveta «Engraulis ringens» y cobia «Rachycentron canadum») en distintas presentaciones (entero, filete, carne molida, congelado). No obstante, en el presente estudio, las muestras de anchoveta (control y tratamiento) desollada, descabezada y eviscerada, congeladas a $-30{ }^{\circ} \mathrm{C}$ y almacenadas a $-25{ }^{\circ} \mathrm{C}$ presentaron un tiempo de vida útil de 14 meses. Al respecto, Semenov et al. (1979) (citado por Karacam y Boran, 1996) indicaron que el periodo de vida útil de los pescados depende de las operaciones previas a la congelación; es decir, recepción, desollado, desangrado, lavado, estibado y congelado. En este estudio, dichas etapas fueron ejecutadas en el mismo lugar de captura y en el menor tiempo posible para mantener la alta frescura de la anchoveta y minimizar su deterioro inicial por oxidación. Sin embargo, en otros estudios, el tiempo demandado en el transporte hasta un centro de investigación probablemente iniciaría y catalizaría el deterioro de las muestras.

Por ello, los ejemplares de anchoveta trabajados (con solo $5 \mathrm{~h}$ de captura) presentaron un lomo de color azulado brillante, indicando una gran frescura. Además, el eficiente desangrado de la anchoveta descabezada y eviscerada en presencia de citrato sódico (con efecto quelante de iones prooxidantes) contribuyó a la prolongación del tiempo de vida útil (Cuadro 6).

\section{Conclusiones}

- El periodo de vida útil, según el análisis sensorial, de la anchoveta desollada, descabezada y eviscerada almacenada a $-25{ }^{\circ} \mathrm{C}$ fue de 14 meses. 
- El periodo de vida útil alcanzado por las muestras fue favorecido por la excelente frescura inicial de la materia prima y rapidez en la ejecución del procesamiento primario hasta su congelación.

- El comportamiento del contenido de hierro hemínico total y la estabilidad de los ácidos grasos poliinsaturados respaldaron la aceptabilidad del análisis sensorial de las muestras.

\section{Agradecimiento}

Los autores agradecen a los profesionales del LABS-ITP por la calidad de servicio mostrado durante la ejecución del estudio y a la empresa pesquera Pezex S.A. por facilitar sus instalaciones durante la producción primaria de los ejemplares de anchoveta en estudio.

\section{Literatura Citada}

1. Ahn D, Wolfe F, Sim J. 1993. Prevention of lipid oxidation in precooked turkey meat patties with packaging and antioxidant combinations. J Food Sci 58: 283-287. doi: 10.1111/ j.1365-2621.1993.tb04256.x

2. Albrecht M, Salas A. 2015. Chemical composition of light and dark muscle of Peruvian anchovy (Engraulis ringens) and its seasonal variation. J Aquat Food Prod Tech 24: 191-196.

3. AOAC Official method 940.28 Fatty acids (free) in crude and refined oils. Titration method. In: AOAC 2005. $18^{\text {th }}$ ed. Ch 41. p 12-13.

4. AOAC Official method 965.33 Peroxide value of oils and fats. Titration method. In AOAC 2005. $18^{\text {th }}$ ed. Ch. 41. p 11-12.

5. Aubourg SP, Ugliano M. 2002. Effect of brine pre-treatment on lipid stability of frozen horse mackerel (Trachurus trachurus). Eur Food Res Technol 215: 91-95. doi: 10.1007/s00217-002-0530-1
6. Bligh E, Dyer W. 1959. A rapid method of total lipid extraction and purification. Can J Biochem Physiol 37: 911-917. doi: 10.1139/059-099

7. Chaijan M, Benjakul S, Visessanguan W, Faustman C. 2005. Changes of pigments and color in sardine (Sardinella gibbosa) and mackerel (Rastrelliger kanagurta) muscle during iced storage. Food Chem 93: 607-617. doi: 10.1016/ j.foodchem.2004.10.035

8. Chávez, J, Ayala M, Albrecht M, Salas A. 2004. Cambios químicos, sensoriales y microbiológicos en perico (Coryphaena hippurus) almacenado en hielo. Bol Inst Tec Pesq 6: 15-21.

9. [FAO] Organización de las Naciones Unidas para la Alimentación y la Agricultura. 2009. Directrices para la inspección del pescado basada en los riesgos. Roma: FAO. Estudio FAO alimentación y nutrición. 99 p.

10. González D, Puig J, Paredes A, Finol H. 2004. Cambios químicos y estructurales en tronquitos de sardina (Sardinella aurita $\mathrm{V}$.) congelados y almacenados a $-40{ }^{\circ} \mathrm{C}$. Rev Científ FCV-LUZ 14 : 303-310.

11. Hamid R, Gheisari H, Moller J, Adams C, Skibsted L. 2010. Sodium chloride or heme protein induced lipid oxidation in raw, minced chicken meat and beef. Czech J Food Sci 28: 364-375.

12. Ibrahim K. 2007. Antimicrobial and antioxidant effects of sodium acetate, sodium lactate and sodium citrate in refrigerated sliced salmon. Food Control 18: 566-575. doi: 10.1016/j.foodcont. 2006.02.002

13. [IMARPE] Instituto del Mar del Perú. 2014. Tallas mínimas y porcentaje de tolerancia máxima de juveniles de recursos hidrobiológicos. [Internet]. Disponible en: http://www.imarpe.pe/imarpe/ tallas_minimas/tallas_minimas.php

14. Karacam H, Boran M. 1996. Quality changes in frozen whole and gutted anchovies during storage at $-18^{\circ} \mathrm{C}$. Int $\mathrm{J}$ Food Sci Tech 31: 527-531. doi: 10.1046/ j.1365-2621.1996.00355.x 
15. Maqsood S, Benjakul S. 2011. Effect of bleeding on lipid oxidation and quality changes of Asian seabass (Lates calcarifer) muscle during iced storage. Food Chem 124: 459-467. doi: 10.1016/ j.foodchem.2010.06.055

16. Maza S, Solari AF Salas A. 2008. Cambios en la calidad de anchoveta entera y HG durante el almacenamiento en congelación. Bol Inst Tec Pesq 8: 37-44.

17. Memon NN, Talpur FN, Sherazi STH, Bhanger MI. 2010. Impact of refrigerated storage on quality of oil from freshwater jarko (Wallago attu) fish. Pak J Anal Environ Chem 11: 37-43.

18. Okoro C, Aboaba O, Babajide O. 2010. Quality assessment of a common Nigerian marine fish, croaker (Pseudotolithus elongatus) under different storage conditions. NY Sci J 3: 29-36.

19. Prevot G, Mordret M. 1976. Utilisation des colonnes capillaires de yerre pour l'analyse des cords gras par chromatographie en phase gazeose. Rev F Corps Gras 23: 7-8.

20. [PRODUCE] Ministerio de la Producción. 2014. Boletín de Anuario Estadístico. $196 \mathrm{p}$.

21. Rezaei M, Hosseini SF. 2008. Quality assessment of farmed rainbow trout (Oncorhynchus mykiss) during chilled storage. J Food Sci 73: 93-96. doi: 10.1111/j.1750-3841.2008.00792.x

22. Richards MP, Hultin HO. 2002. Contributions of blood components to lipid oxidation in fish muscle. J Agric Food Chem 50: 555-564.

23. Rule DC, Broughton KS, Shellito SM, Maiorano G. 2002. Comparison of muscle fatty acid profiles and cholesterol concentrations of bison, beef cattle, elk and chicken. J Anim Sci 80: 1202-1211.

24. Salas A, Barriga M, Albrecht M, Castro R. 2004. Estabilización oxidativa de la pulpa de jurel (Trachurus murphyi) mediante el uso de antioxidantes. Bol Inst Tec Pesq 6: 9-14.

25. Salas A, Maza S, Barriga M. 2007. Efecto de antioxidantes sobre la estabi- lidad de la pulpa de anchoveta durante el almacenamiento en congelación. Bol Inst Tec Pesq 8: 75-83.

26. Salvay A, Grigera R, Colomboy $M$. 2003. The role of hydration on the mechanism of allosteric regulation: in situ measurements of the oxygen-linked kinetics of water binding to hemoglobin. Biophys J 84: 564-570. doi: 10.1016/ S0006-3495(03)74876-0

27. Sánchez J, Sosa J, Chávez J. 2004. Manipuleo y conservación de la anchoveta peruana para consumo humano directo. Bol Inst Tec Pesq 6: 39-46.

28. Schricker B, Miller D. 1983. Effects of cooking and chemical treatment on heme and nonheme iron in meat. J Food Sci 48: 1340-1343. doi: 10.1111/j.13652621.1983.tb09225.x

29. Taheri S, Motalebi AA, Fazlara A. 2012. Antioxidant effect of ascorbic acid on the quality of cobia (Rachycentron canadum) fillets during frozen storage. Iranian J Fish Sci 11: 666-680.

30. Terayama M, Yamanaka H. 2000. Effect of bleeding on the quality of skipjack. Nippon Suisan Gakk 66: 852858. doi: 10.2331/suisan.66.852

31. Thiansilakul Y, Benjakul S, Richards M. 2010. Changes in heme proteins and lipids associated with off-odour of seabass (Lates calcarifer) and red tilapia (Oreochromis mossambicus x $O$. niloticus) during iced storage. Food Chem 121: 1109-1119. doi: 10.1016/ j.foodchem.2010.01.058

32. Turhan S, Sule N, Bogachan T. 2004. Effect of cooking methods on total and heme iron contents of anchovy (Engraulis encrasicholus). Food Chemistry 88: 169-172. doi: 10.1016/ j.foodchem.2004.01.026

33. Vyncke W. 1975. Evaluation of the direct thiobarbituric acid extraction method for determining oxidative rancidity in mackerel (Scomber scombrus L.). Eur J Lip Sci Tech 77: 239-240. doi: 10.1002/lipi.19750770610 